\title{
Does Anesthesiologist-Directed Sedation Afford Superior Deep Cannulation Rates and Procedural Outcomes for ERCP in the Community Setting?
}

\author{
Brad Bowyer ${ }^{1,2}$, Kathy Geissler ${ }^{1}$, Robert Barclay ${ }^{1,2}$, Sumeet Tewani, ${ }^{1,2}$, James Frakes ${ }^{2}$, \\ Nicholas Brown'2, Matthew Houlihan1, Kunal Patel2, Andrew Spiel² \\ ${ }^{1}$ Rockford Gastroenterology Associates, Rockford, IL, USA \\ ${ }^{2}$ University of Illinois College of Medicine Rockford, Rockford, IL, USA \\ Email: "drbowyer@rockfordgi.com
}

Received 13 January 2016; accepted 23 February 2016; published 26 February 2016

Copyright (C) 2016 by authors and Scientific Research Publishing Inc.

This work is licensed under the Creative Commons Attribution International License (CC BY). http://creativecommons.org/licenses/by/4.0/

(c) $\underset{\mathrm{EY}}{\mathrm{F}}$ Open Access

\section{Abstract}

Aim: To compare outcomes by sedation class in community patients undergoing index endoscopic retrograde cholangiopancreatography (ERCP). Methods: Nineteen hundred sixteen consecutive patients underwent ERCP from May 2005 to May 2011. Eight hundred thirty seven patients were excluded due to prior papillary intervention or attempted ERCP. A total of 1079 patients were included. The 981 patients who underwent gastroenterologist directed sedation (GDS) served as the control population, while the 98 patients who received anesthesiologist directed sedation (ADS) served as the case population. Medical records were analyzed for patient demographics, procedure indication, adverse events, case complexity, procedural failure and sedation failure. Case complexity was defined by the grading system proposed by the working party of the ASGE Quality Committee. Sedation failure was defined by agitation or airway compromise prompting termination of the ERCP. Reasons for procedural failure included surgically altered anatomy, luminal obstruction, and technical failure. Study endpoint was defined as successful deep cannulation of the intended target duct. Results: Demographic distribution did not differ between the GDS and the ADS groups. Cannulation success rates were similar between the two groups, with $89.85 \%$ in the GDS group, and $89.58 \%$ in the ADS group $(P=0.864)$. There were no statistical differences between sedation groups in procedural or respiratory adverse events. Technical failure was the predominant basis for deep cannulation failure in both groups. Agitation and airway compromise accounted for deep cannulation failure similarly in both groups. The need for reversal agents was low but similar in both groups. There was no statistical advantage in deep cannulation success rate by complexity grade in either sedation class. Conclusion: Excellent procedural outcomes and

How to cite this paper: Bowyer, B., Geissler, K., Barclay, R., Tewani, S., Frakes, J., Brown, N., Houlihan, M., Patel, K. and Spiel, A. (2016) Does Anesthesiologist-Directed Sedation Afford Superior Deep Cannulation Rates and Procedural Outcomes for ERCP in the Community Setting? Open Journal of Gastroenterology, 6, 46-52.

http://dx.doi.org/10.4236/ojgas.2016.62007 
low adverse event rates were achieved using GDS, a more accessible and cost-effective method in a community-based setting.

\title{
Keywords
}

\author{
ERCP, Community-Based Medicine, Conscious Sedation, Anesthesiologist-Directed Sedation, \\ Gastroenterologist-Directed Sedation
}

\section{Introduction}

Endoscopic retrograde cholangiopancreatography (ERCP) has evolved into a complex procedure involving specialized operators, equipment and supporting personnel. ERCP was first described in 1965 as a novel method for imaging the pancreatic duct and biliary tree [1]. Successful management of choledocholithiasis with biliary sphincterotomy was described in 1974 by Classen [2] in Germany and Kawai [3] in Japan. Advances in computerized axial tomography (CT) body imaging, endoscopic ultrasound (EUS), and magnetic resonance cholangiopancreatography (MRCP) have been essential in the evolution of ERCP applications from diagnostic to therapeutic. By 2007, estimated 500,000 ERCPs were performed annually in the US. ERCP utilization has steadily increased over time [4], with current annual US ERCP volumes expected to exceed those published in 2007 [5]. Analysis of the 2006 National Inpatient Sample Database for US ERCPs found that 90 percent were performed in centers with volumes of less than 200 per year, suggesting a large portion of ERCPs during the study period were performed in a community setting [6].

Despite the nearly 50-year history and its widespread use, there remain a number of areas of uncertainty regarding the optimal performance of ERCP. One such area pertains to the method of procedural sedation. ERCP is often technically demanding and more prolonged with higher associated rates of adverse events as compared to other gastrointestinal endoscopic procedures [7]. ERCP is preferentially performed with the patient in a prone position to improve papillary access and optimal fluoroscopic imaging; however, this poses a challenge in airway management and assessment of respiratory status while providing the appropriate continuum of sedation necessary to perform the procedure. Therefore, some authors have recommended that deep, anesthesiologist directed sedation (ADS) be used routinely or preferentially for ERCP [8]-[10]. No guidelines for use of ADS exist, however, and presumed superiority of ADS is not supported by available evidence [11]. This is concerning to gastroenterologists in community settings, where anesthesia access is a limited resource that can add significantly to delays in patient care and patient costs. In our group's experience, gastroenterologist-directed sedation (GDS) for ERCP is safe and effective. Furthermore, we feel that recommendations for ADS for ERCP do not sufficiently address drawbacks such as additional cost, availability, and unforeseen risks. Therefore, we sought to compare outcomes of GDS versus ADS in our community-based patient population undergoing index ERCP.

\section{Materials and Methods}

The study protocol was designed as a retrospective review of existing records from patients within our practice who previously underwent index ERCP. The protocol was reviewed and approved with Exempt Status by the Institutional Review Board of the University Of Illinois College Of Medicine (Rockford, IL). We reviewed Rockford Gastroenterology Associates patient records from May 2005 through May 2011. A total of 1916 patients underwent ERCP by 8 experienced, board certified gastroenterologists from Rockford Gastroenterology Associates, Ltd. Upon review, 837 of these patients were excluded due to prior papillary intervention or attempted ERCP.

All of the procedures were performed by board certified gastroenterologists or anesthesiologists. The majority of GDS cases were performed by 4 endoscopists at any one time over the 6 years of the study with an average 275 ERCP's performed per year by those providers. More recent records were excluded from analysis due to changes in personnel that added delays in data collection and document preparation.

The ADS group served as the cases and the GDS group served as the controls. We anticipated that the ADS group comprised $10 \%$ of all ERCPs performed. Previous studies have established that successful cannulation should be achieved in $90 \%$ of patients if the papilla is reached [12]. This study was powered to detect a $10 \%$ 
lower successful cannulation rate in the GDS group as compared to the ADS group. For a case-control study with an alpha level of $5 \%$, power of $80 \%$, and 1:9 case-control ratio, the sample size required 1050 patients. A p value of 0.05 was considered to represent a statistically significant difference between the two groups. Patient demographics, clinical data, and outcomes were analyzed. Comparisons between the groups utilized the Student t-test for continuous variables and the Chi-square or Fisher exact tests as indicated for categorical variables.

Inpatient and outpatient medical records of the remaining 1079 patients were analyzed for demographics, procedure indications, deep cannulation success, sedation adverse events (agitation and airway compromise), and procedural adverse events (pancreatitis, perforation, cholangitis, hemorrhage, and death). Sedation failure was defined by premature termination of the procedure related to agitation or airway compromise. Anatomic failure was defined by inability to achieve cannulation of the desired duct despite adequate papillary access, related to surgically altered anatomy or luminal obstruction. Deep cannulation failure was defined as technical failure if papillary access was achieved but the desired duct was not deeply cannulated and opacified with contrast. ERCP complexity was determined according to the grading system proposed by the working party of the ASGE Quality Committee [13]. ERCP adverse events were assigned using the consensus statement published by Cotton et al. [14].

\section{Results}

Table 1 summarizes our population demographics. Of 1079 patients included in this study, 98 received ADS (9.1\%) and 981 received GDS (90.9\%). Of the 98 patients in the ADS group, 42 were done with Monitored Anesthesia Care (MAC) and 56 with general anesthesia. Demographic distribution did not differ between the two groups. Deep Cannulation success rates were adjusted to exclude patients in whom papillary access was not achieved. Cannulation success rates were similar between the two groups, with $89.85 \%(850 / 946)$ in the GDS group, and $89.58 \%(86 / 96)$ in the ADS group $(P=0.864)$. ERCP complexity (Table 1$)$ was not significantly different between GDS and ADS patients $(P=0.082)$. Table 2 outlines Indication for procedure. Table 3 outlines reasons for deep cannulation failure. Technical failure was the predominant basis for deep cannulation failure in both groups at $6.01 \%$ in the GDS group and $6.12 \%$ in the ADS group, respectively $(P=1.000)$. Sedation failure was the same in both groups $(P=1.000)$. Deep cannulation failure occurred due to agitation in 15 GDS patients and 1 ADS patient $(P=1.000)$, and occurred due to airway compromise in 5 GDS patients and 1 ADS patient $(P=0.436)$. Reversal agents were necessary in 6 GDS patients and 1 ADS patient $(P=0.488)$. Similarly, rates of pancreatitis, cholangitis, and hemorrhage were virtually identical between the 2 groups $[P=$ 1.000 for each (Table 4)]. There were 6 perforations in the GDS group and 1 in the ADS group $(P=0.488)$. There was 1 death and 1 reported case of aspiration, both in the ADS group $(P=0.091)$. There was no statistical advantage in deep cannulation success rate by complexity grade in either sedation class. Similarly, there were no differences in adverse event rates by ERCP complexity grade between the GDS and ADS groups.

\section{Discussion}

This study analyzed a large population of patients who underwent ERCP by experienced biliary endoscopists in a community setting. The indications for ERCP were similar to those reported in other population-based studies [15] [16]. The focus of the current study was on the type of sedation used for ERCP, specifically whether standard use of GDS was adequate to achieve procedural success and equivalent to outcomes achieved in ERCPs conducted under ADS. Due to the retrospective design of our study, it is unclear in the majority of cases (63\%) why the endoscopist chose ADS over standard practice GDS. When documented, the reasons for ADS included prior history of sedation failure $(15 \%)$, alcohol or narcotic use $(10 \%)$, pre-existing respiratory issues $(6 \%)$, pregnant or post-partum status $(3 \%)$ and morbid obesity $(2 \%)$. Underlying co-morbidities were not consistently documented in the medical records and therefore the American Society of Anesthesiologist (ASA) classification was not utilized. Although one could speculate that patients for whom ADS was selected at the outset were more likely to have a greater degree of procedural complexity, our observations did not support this, as the complexity based on the Cotton scale was similar for both sedation groups (Table 1). We identified high rates of procedural success and low rates of significant adverse events in both groups. A recent multinational study of 13,018 patients by Peng et al. found that general anesthesia was associated with greater cannulation success only in the $6.7 \%$ of patients who required pre-cut sphincterotomy for biliary access [17]. Our study did not specifically address pre-cut sphincterotomy. The size of our anesthesia cohort and infrequent necessity of this technique would 
not allow for meaningful statistical analysis on this subset of patients. However, our cannulation rate of $89.9 \%$ was comparable to the $89.8 \%$ non-pre-cut population as reported by Peng et al.

Our results challenge recent recommendations that all patients undergoing ERCP require deep sedation or general anesthesia (8). Similar to other smaller studies [18]-[20] excellent, equivalent procedural outcomes and low adverse event rates were achieved by our group in both the GDS and ADS patients. Given these findings

Table 1. Patient Demographics.

\begin{tabular}{|c|c|c|c|}
\hline & GDS & ADS & \\
\hline Total Patients & 981 & 98 & \\
\hline Male & $392(39.96 \%)$ & $46(46.94 \%)$ & $P=0.196$ \\
\hline Female & $589(60.04 \%)$ & $52(53.06 \%)$ & \\
\hline Average Age & 61.7 & 53.95 & \\
\hline Papillary Access Achieved & 946 & 96 & \\
\hline Deep Cannulation Success & $850(89.85 \%)$ & $86(89.58 \%)$ & $P=0.861$ \\
\hline $\begin{array}{l}\text { Cotton Complexity } 1 \\
\text { Deep cannulation of duct of interest, main papilla, sampling Biliary stent } \\
\text { removal/exchange }\end{array}$ & $13.05 \%$ & $17.35 \%$ & $P=0.082$ \\
\hline $\begin{array}{l}\text { Cotton Complexity } 2 \\
\text { Biliary stone extraction }<10 \mathrm{~mm} \text {, treat biliary leaks, treat extrahepatic strictures, place } \\
\text { prophylactic pancreatic stents }\end{array}$ & $72.48 \%$ & $61.22 \%$ & \\
\hline $\begin{array}{l}\text { Cotton Complexity } 3 \\
\text { Biliary stone extraction }>10 \mathrm{~mm} \text {, minor papilla cannulation in divisum, and therapy, } \\
\text { remove internally migrated biliary stents, intraductal imaging, biopsy, FNA, manage } \\
\text { acute or recurrent pancreatitis, treat pancreatic strictures, remove pancreatic stones } \\
\text { mobile and }<5 \mathrm{~mm} \text {, treat hilar tumors, treat benign biliary structures, hilum and above, } \\
\text { manage suspected sphincter of Oddi dysfunction }\end{array}$ & $13.97 \%$ & $21.43 \%$ & \\
\hline $\begin{array}{l}\text { Cotton Complexity } 4 \\
\text { Remove internally migrated pancreatic stents, intraductal image-guided therapy, } \\
\text { pancreatic stones impacted and/or }>5 \mathrm{~mm} \text {, Intrahepatic stones, pseudocyst drainage, } \\
\text { necrosectomy, ampullectomy, ERCP after Whipple or Roux-en-Y }\end{array}$ & $0.51 \%$ & $0.0 \%$ & \\
\hline
\end{tabular}

Table 2. Procedural Indications.

\begin{tabular}{ccc}
\hline & GDS & ADS \\
\hline Abnormal Imaging & $85(9 \%)$ & $8(8 \%)$ \\
Abnormal Liver Function & $18(2 \%)$ & $12(12 \%)$ \\
Jaundice & $244(25 \%)$ & $18(18 \%)$ \\
Chronic or Recurrent Pancreatitis & $161(16 \%)$ & $20(20 \%)$ \\
Bile Leak & $37(4 \%)$ & $4(4 \%)$ \\
Stone or Suspected Stone & $400(41 \%)$ & $32(32 \%)$ \\
Tumor or Stricture & $36(4 \%)$ & $4(4 \%)$ \\
\hline
\end{tabular}

Table 3. Reasons for Cannulation Failure.

\begin{tabular}{|c|c|c|c|}
\hline & GDS & ADS & $P$-Value \\
\hline Total Patients & 981 & 98 & \\
\hline Inability to Access Papilla for Cannulation Attempt & 35 & 2 & $P=0.570$ \\
\hline Papillary Access Achieved & 946 & 96 & $P=0.998($ Overall $)$ \\
\hline Sedation Failure & $20(2.04 \%)$ & $2(2.04 \%)$ & $P=1.000$ \\
\hline Anatomic Failure & $17(1.73 \%)$ & $2(2.04 \%)$ & $P=0.688$ \\
\hline Technical Failure & $59(6.01 \%)$ & $6(6.12 \%)$ & $P=1.000$ \\
\hline
\end{tabular}


Table 4. Adverse Events.

\begin{tabular}{cccc}
\hline Adverse Events & GDS & ADS & $P$-Value \\
\hline Pancreatitis & $34(3.15 \%)$ & $3(3.06 \%)$ & $P=1.000$ \\
Cholangitis & $5(0.46 \%)$ & $0(0 \%)$ & $P=1.000$ \\
Hemorrhage & $2(0.18 \%)$ & $0(0 \%)$ & $P=1.000$ \\
Perforation & $6(0.56 \%)$ & $1(1.02 \%)$ & $P=0.488$ \\
Death & $0(0 \%)$ & $1(1.02 \%)$ & $P=0.091$ \\
Aspiration & $0(0 \%)$ & $1(1.02 \%)$ & $P=0.091$ \\
\hline
\end{tabular}

supportive of the use of gastroenterologist-directed sedation for ERCP, the promulgation of policies that endorse anesthesiologist-directed sedation for ERCP seems unjustified. Indeed, one may question the unintended consequences of promoting such an approach in the absence of firm evidence of superior outcomes from prospective randomized studies. For example, some institutions may not have ready access to anesthesia services but may otherwise be equipped to perform ERCP using conventional sedation. In such a scenario, an "anesthesia-only" approach could lead to delay in the performance of ERCP and poorer outcomes. Additionally, we have found that when anesthesiologists are involved with sedation for ERCP, general anesthesia is preferentially employed. General anesthesia carries unique risks, such as tracheo-laryngeal injury [21], that are not associated with moderate sedation. Recent data have raised concerns about adverse respiratory sequelae of deep sedation for endoscopic procedures [22]. Finally, cost consciousness remains relevant in the practice of evidence based medicine. Increased number of endoscopic procedures and increasing use of anesthesia for such procedures is estimated to add billions of dollars to the cost of endoscopic procedures [23].

Deep sedation directed by an anesthesiologist was necessary in only a small subset of our patients undergoing ERCP. The prospect of universal adoption of anesthesiologist-directed sedation is an appealing convenience that allows the gastroenterologist to focus only on the procedural aspects of ERCP. ADS is certainly justified in cases where conventional sedation has failed or case complexity predicts procedure length beyond the therapeutic window of conventional sedation. Teaching centers certainly may default to anesthesiologist-directed sedation to facilitate supervision of physicians in training. However, in our experience as community based gastroenterologists, universal adoption of anesthesiologist-directed sedation is not warranted or possible in the absence of more robust anesthesia support services.

As timely anesthesia support is not consistently available, our group has been exploring the use of a novel alternative agent, dexmedetomidine, as an adjunct to GDS [24]. Our preliminary experience has been favorable, but further study comparing this novel sedation approach with ADS is required to determine if this will prove to be a suitable alternative when anesthesia services are not readily available.

The authors acknowledge limitations inherent in a single site, non-randomized, retrospective study. Training and experience of the anesthesiologists in the ADS group were not considered in our analysis as they represented a small sample drawn from a large pool of board certified anesthesiologists in our hospitals. In addition, data on underlying patient co-morbidities was not analyzed. Brief intraprocedural events which did not prematurely terminate the procedure, such as bradycardia, hypotension and hypoxia were excluded from analysis.

Accurate sedation choice remains inexact. Our findings suggest additional studies are required to identify patient-specific or procedure-related factors which would more readily stratify patients into particular sedation groups rather than adopting universal anesthesiologist-directed sedation as the preferential method for ERCP.

\section{Conclusion}

Excellent procedural outcomes and low adverse event rates were achieved in our practice using GDS. These outcomes were statistically indistinguishable from ADS. The promulgation of policies that recommend anesthesiologist-directed sedation preferentially over conventional gastroenterologist-directed sedation for ERCP is not supported by our findings.

\section{Conflict of Interests}

The authors have no conflict of interest to disclose. 


\section{References}

[1] Rabinov, K.R. and Simon, M. (1965) Peroral Cannulation of the Ampulla of Vater for Direct Cholangiography and Pancreatography: Preliminary Report of a New Method. Radiology, 85, 693-697. http://dx.doi.org/10.1148/85.4.693

[2] Classen, M. and Demling, L. (1974) Endoskopische sphinkterotomie der papilla vateri und steinextracktion aus dem ductus choledochus. Deutsche Medizinische Wochenschrift, 99, 496-497. http://dx.doi.org/10.1055/s-0028-1107790

[3] Kawai, K., Akasaka, Y., Murakami, K., Tada, M., Kohli, Y. and Nakajima, M. (1974) Endoscopic Sphincterotomy of the Ampulla of Vater. Gastrointestinal Endoscopy, 20, 148-151. http://dx.doi.org/10.1016/S0016-5107(74)73914-1

[4] Coelho-Prabhu, N., Shah, N., Houten, H., Kameth, P.S. and Baron, T.H. (2013) Endoscopic Retrograde Cholangiopancreatography: Utilisation and Outcomes in a 10-Year Population-Based Cohort. BMJ Open, 3, Article ID: e002689. http://dx.doi.org/10.1136/bmjopen-2013-002689

[5] Andriulli, A., Silvano, L., Napolitano, G., Niro, G., Valvano, M.R., Spirito, F., Pilotto, A. and Forlano, R. (2007) Incidence Rates of Post-ERCP Complications: Asystematic Survey of Prospective Studies. The American Journal of Gastroenterology, 102, 1781-1788. http://dx.doi.org/10.1111/j.1572-0241.2007.01279.x

[6] Varadarajulu, S., Kilgore, M., Wilcox, C. and Eloubeidi, M.A. (2006) Relationship among Hospital Volume, Length of Stay, and Technical Outcomes. Gastrointestinal Endoscopy, 64, 338-347. http://dx.doi.org/10.1016/j.gie.2005.05.016

[7] Cotton, P. and Frakes, J.T. (2013) Medicolegal Issues in ERCP. In: Baron, T.H., Kozarek, R. and Carr-Locke, D.L., Eds., A Practical Guide to ERCP, Elsevier Ltd., London, 92-102.

[8] Kachaamy, T.A. and Faigel, D.O. (2013) Improving ERCP Quality and Decreasing Risk to Patients and Providers. Journal of Gastroenterology and Hepatology, 7, 531-540. http://dx.doi.org/10.1586/17474124.2013.824703

[9] Martindale, S.J. (2006) Anaesthetic Considerations during Endoscopic Retrograde Cholangiopancreatography. Anaesthesia and Intensive Care, 34, 475-480.

[10] Lordan, J., Woods, J., Keeling, P. and Paterson, I.M. (2011) A Retrospective Analysis of Benzodiazepine Sedation vs. Propofol Anaesthesia in 252 Patients Undergoing Endoscopic Retrograde Cholangiopancreatography. Hepato Pancreato Biliary, 13, 174-177. http://dx.doi.org/10.1111/j.1477-2574.2010.00266.x

[11] Garewal, D., Powell, S., Milan, S.J., Nordmeyer, J. and Waikar, P. (2012) Sedative Techniques for Endoscopic Retrograde Cholangiopancreatography. Cochrane Database of Systematic Reviews, 6, Article ID: CD007274. http://dx.doi.org/10.1002/14651858.cd007274.pub2

[12] Chutkan, R., Ahmad, A., Cohen, J., et al. (2006) American Society for Gastrointestinal Endoscopy. ASGE Training Committee. ERCP Core Curriculum. Gastrointestinal Endoscopy, 63, 361-376. http://dx.doi.org/10.1016/j.gie.2006.01.010

[13] Cotton, P.B., Eisen, G., Romagnuolo, J., et al. (2011) Grading the Complexity of Endoscopic Procedures: Results of an ASGE Working Party. Gastrointestinal Endoscopy, 73, 868-874.

[14] Cotton, P.B., Lehman, G., Vennes, J., Geenen, J.E., Russell, R.C.G., Meyers, W.C., Liguory, C. and Nicki, N. (1991) Endoscopic Sphincterotomy Complications and Their Management: An Attempt at Consensus. Gastrointestinal Endoscopy, 37, 383-393. http://dx.doi.org/10.1016/S0016-5107(91)70740-2

[15] Mehta, P.P., Vargo, J.J., Dumot, J.A., Parsi, M.A., Lopez, R. and Zuccaro, G. (2011) Does Anesthesiologist-Directed Sedation for ERCP Improve Deep Cannulation and Complication Rates? Digestive Diseases and Sciences, 56, 21852190. http://dx.doi.org/10.1007/s10620-011-1568-3

[16] Raymondos, K., Panning, B., Bachem, I., Manns, M.P., Piepenbrock, S. and Meier, P.N. (2002) Evaluation of Endoscopic Retrograde Cholangiopancreatography under Conscious Sedation and General Sedation. Endoscopy, 34, 721726. http://dx.doi.org/10.1055/s-2002-33567

[17] Peng, C., Nietert, P., Cotton, P., et al. (2013) Predicting Native Papilla Biliary Cannulation Success Using a Multinational Endoscopic Retrograde Cholangiopancreatography (ERCP) Quality Network. BMC Gastroenterology, 13, 147. http://dx.doi.org/10.1186/1471-230X-13-147

[18] Kongkam, P., Rungsun, R., Punyathavorn, S., et al. (2008) Propofol Infusion versus Intermittent Meperidine and Midazolam Injection for Conscious Sedation in ERCP. Journal of Gastrointestinal and Liver Diseases, 23, 292-297.

[19] Qadeer, M., Vargo, J., Khandwala, F., Lopez, R. and Zuccaro, G. (2005) Propofol versus Traditional Sedative Agents for Gastrointestinal Endoscopy: A Meta-Analysis. Clinical Gastroenterology and Hepatology, 3, 1049-1056. http://dx.doi.org/10.1016/S1542-3565(05)00742-1

[20] Chawla, S., Katz, A., Attar, B. and Go, B. (2013) Endoscopic Retrograde Cholangiopancreatography under Moderate Sedation and Factors Predicting Need for Anesthesiologist Directed Sedation: A County Hospital Experience. World Journal of Gastrointestinal Endoscopy, 5, 160-164. http://dx.doi.org/10.4253/wjge.v5.i4.160

[21] Weber, S. (2002) Traumatic Complications of Airway Management. Anesthesiology Clinics of North America, 20, 503512. http://dx.doi.org/10.1016/S0889-8537(02)00014-7

[22] Cooper, G., Kou, T. and Rex, D. (2013) Complications Following Colonoscopy with Anesthesia Assistance. JAMA In- 
ternal Medicine, 173, 551-556. http://dx.doi.org/10.1001/jamainternmed.2013.2908

[23] Liu, H., Waxman, D.A., Main, R. and Mattke, S. (2012) Utilization of Anesthesia Services during Outpatient Endoscopies and Colonoscopies and Associated Spending in 2003-2009. JAMA Internal Medicine, 307, 1178-1184. http://dx.doi.org/10.1001/jama.2012.270

[24] Foster, B., Geissler, K., Solverson, P., Thukral, C., Barclay, R.L. and Vicari, J.J. (2015) SU1151 Dexmedetomidine for GI Procedural Sedation: Initial Experience in a Community-Based Practice. Gastrointestinal Endoscopy, 81, AB310AB311. http://dx.doi.org/10.1016/j.gie.2015.03.472 